\title{
Influencia del Índice de Masa Corporal en las Características Faciales de Mujeres Jóvenes. Resultados Preliminares
}

\author{
Body Mass Index Influence in Facial Pattern of Young Women. Preliminary Results
}

\author{
Claudio Huentequeo-Molina ${ }^{*, * *}$; Pablo Navarro" ${ }^{* * *}$ \& Sergio Olate ${ }^{*, *, * * * *}$
}

HUENTEQUEO-MOLINA, C.; NAVARRO, P.; OLATE, S. Influencia de índice de masa corporal en las características faciales de mujeres jóvenes. Resultados preliminares. Int. J. Odontostomat., 7(3):407-414, 2013.

RESUMEN: La posición y volumen de tejidos blandos de la cara generan la necesidad de una planificación precisa al momento de cirugias faciales; la influencia del tejidos adiposo en estas caracteristicas puede estar presente. El objetivo de este estudio es identificar las medidas volumétricas y no volumétricas de puntos antropométricos faciales y establecer su relación con el índice de masa corporal (IMC). Se analizaron 29 sujetos del sexo femenino de entre 20 y 25 años de edad (promedio de 22,5 años). Se incluyeron sujetos con auto percepcion de normalidad facial y sin condiciones previas asociadas a trauma, cirugia facial u ortodoncia. Se realizó una secuencia de analisis facial realizando medidas con hilo sobre la cara y con pie de metro en los mismos puntos, estudiando distancias entre puntos antropometricos previamente definidos. Inicialmente se realizo el estudio de peso y talla de los sujetos mediante condiciones estandarizadas. En la tercera etapa se realizaron radiografías laterales en cada sujeto realizando mediciones sobre la radiografia para identificar el tejido blando presente en la región labio superior e inferior, punto A, punto $B$ y pogonion. El analisis de datos se realizo con t test de muestras relacionadas, considerando un valor de $p \leq 0.05$; el análisis estadístico ANOVA se utilizó para definir la influencia del IMC sobre las variables estudiadas. El peso obtenido registró un promedio de $57,6 \mathrm{~kg}( \pm 8,3 \mathrm{~kg})$ y la talla presento un promedio de 1,6mt; el IMC obtenido fue de 22,5, registrándose 2 sujetos considerados en peso inferior al normal, 20 con peso normal, 6 en sobrepeso y 1 con obesidad. Se observo que las variables volumetricas fueron diferentes a las variables no volumetricas de la cara y se asociaron significativamente con el IMC. EI IMC no presento relación positiva con las mediciones realizadas en radiografias laterales; finalmente el IMC tambien no se asocio a la auto percepcion de estética facial $(p=0.069)$. Se concluye que el IMC influencia las mediciones obtenidas en la cara y que no influye en los registros realizados en radiografías laterales.

PALABRAS CLAVE: Î́ndice de masa corporal, Estética facial, Cirugía facial.

\section{INTRODUCCIÓN}

La sistematización del análisis facial ha sido estudiado por algunos autores (Farkas et. al, 1985; Starck \& Epker, 1996) donde la idea de proporción y simetría está claramente establecida. Condicionantes quirúrgicas de la cara exigen un estudio profundo a fin de mantener los cánones faciales dentro los rangos aceptables (Farkas et al., 2005; Husein et al., 2010; Uysal et al., 2012). Algunos aspectos recientes con análisis tridimensional y el uso de software han optimizado las técnicas diagnosticas y el manejo quirúrgico de algu- nos pacientes (Dong et al., 2010; Olszewski et al. 2007). Sin embargo, la falta de protocolos y patrones de estudio hacen complejos los análisis comparativos entre diferentes grupos de individuos (Douglas, 2004).

En la planificación de modificaciones faciales, el movimiento de estructura ósea y dentaria son altamente predecibles, sin embargo, la predicción de movimiento de tejidos blandos es aun insuficiente para reconocer con seguridad los desplazamientos facia-

\footnotetext{
Programa de Magister en Odontología, Universidad de La Frontera, Chile.

* Unidad de Cirugía Oral y Maxilofacial, Universidad de La Frontera, Chile.

*** Depto. de Matemáticas y Estadística, Facultad de Ingeniería, Ciencias y Administración, Universidad de la Frontera, Temuco, Chile.

**** Centro de Investigación en Ciencias Biomédicas, Universidad Autónoma de Chile, Chile.
} 
les (Godt et al., 2013; Reddy et al., 2011), pudiendo incluso generar cambios faciales poco deseados y fuera de lo proyectado. De esta forma, los análisis de tejidos blandos de la cara debe ser detalladamente estudiado (Sahni D et al., 2008).

En condiciones normales, el plano adiposo es capaz de variar de forma agresiva la morfología corporal; a pesar de ello, muchas veces no es considerado en la planificación quirúrgica facial (De Greef et al., 2009). De esta forma, algunos autores han señalado que evaluar la cantidad de masa corporal podría beneficiar los análisis y una forma simple de realizarlo seria a través del estudio del índice de masa corporal (IMC) (Codinha et al., 2009; Sipahioglu et al., 2012).

Así considerado, la relación entre tejidos duros y tejidos blandos podrían ser influenciados por la cantidad de tejido adiposo de forma que la gran variabilidad de volumen y forma del tejido blando debe ser considerado en la planificación quirúrgica (Alcalde et al., 2000; Gassmann et al., 1989). El objetivo de esta investigación es determinar la relación entre la morfología de tejidos blandos de la cara junto a su relación con el índice de masa corporal en mujeres de 20 a 25 años de edad.

\section{MATERIAL Y MÉTODO}

Se realizó un estudio descriptivo transversal e inferencial, donde se seleccionó a 29 mujeres chilenas de entre 20 y 25 años de edad. Fueron incluidas estudiantes universitarias que se auto-consideraban con características faciales normales y que accedieron de forma voluntaria al estudio para lo cual firmaron un consentimiento informado accediendo a la investigación; todas las personas que accedieron al estudios completaron una encuesta donde se auto definían como normales en lo referentes a las características faciales; ninguna de ellas se considero anormal o con alteraciones faciales. Se excluyeron mujeres embarazadas, con presencia de enfermedades siquiátricas, con antecedentes de trauma facial que afectaran la morfología de la cara, presencia actual o pasada de tratamiento de ortodoncia, cirugía plástica facial o maxilofacial y mujeres con perdida o agenesia dentaria (no incluidos los terceros molares).

El peso fue registrado dos veces con 5 minutos de diferencia entre cada registro a través de la báscula marca Soehnle $\AA$, modelo Body control Signal F3, con precisión de $100 \mathrm{~g}$. La talla del sujeto fue obtenida con regla rígida milimetrada marca Artel $\circledast$, instalada en un muro perpendicular al piso. El individuo fue ubicado con pies descalzos, apoyado en el muro con la regla instalada en el muro; una escuadra cayo en ángulo recto con la regla del muro, llegando al primer contacto con el cuero cabelludo, registrando el valor en centímetros como la talla del sujeto; esta medición fue realizada en dos oportunidades. Todos los registros fueron realizados entre las $9: 30 \mathrm{~h}$ y las $11: 30 \mathrm{~h}$. Con estos resultados fue obtenido el IMC en base a la fórmula.

$$
\mathrm{IMC}=\frac{\text { masa del individuo }}{\text { altura del individuo }}
$$

Fórmula Matemática utilizada para obtener el Índice de Masa Corporal (IMC) según OMS (http:// apps.who.int/bmi/index.jsp?introPage=intro_3.html)

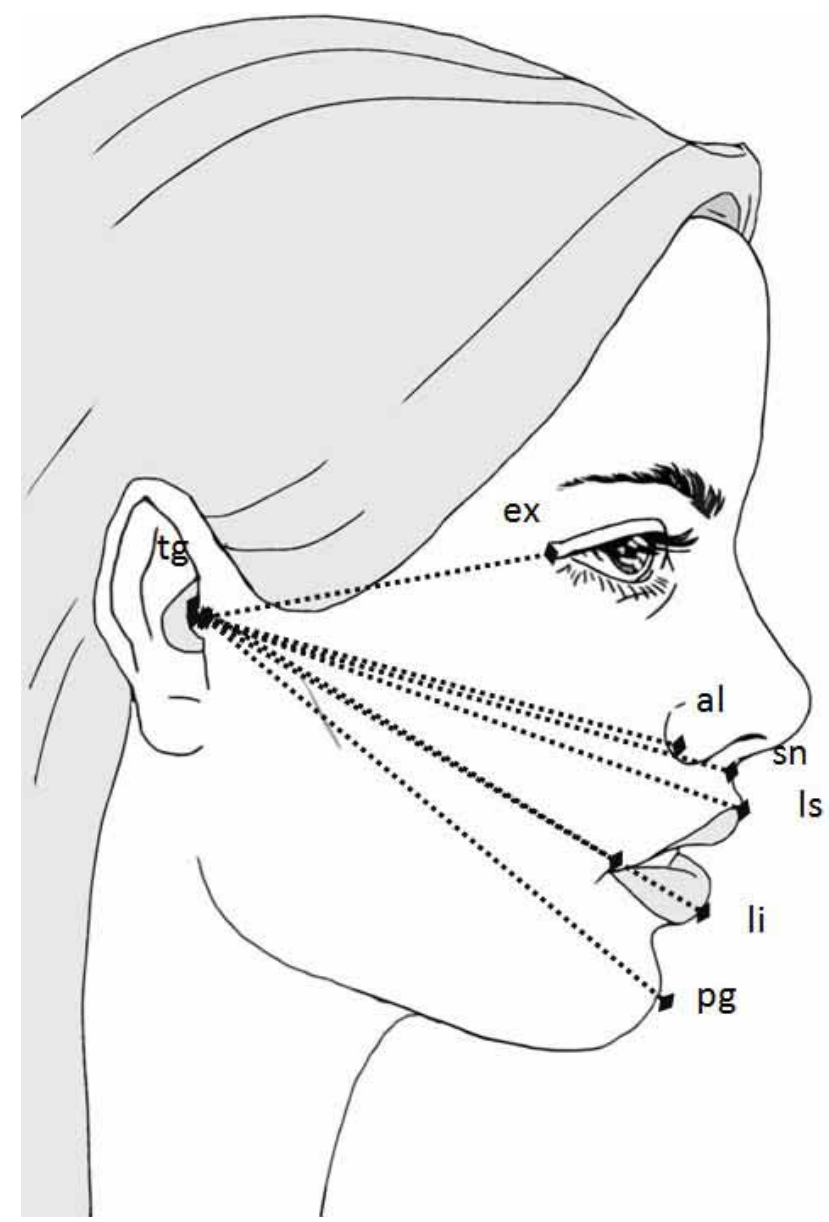

Fig. 2. Ubicación de los puntos antropométricos utilizados en el análisis facial de los 29 individuos, realizando mediciones rectas con pie de metro (medidas no volumétricas). 


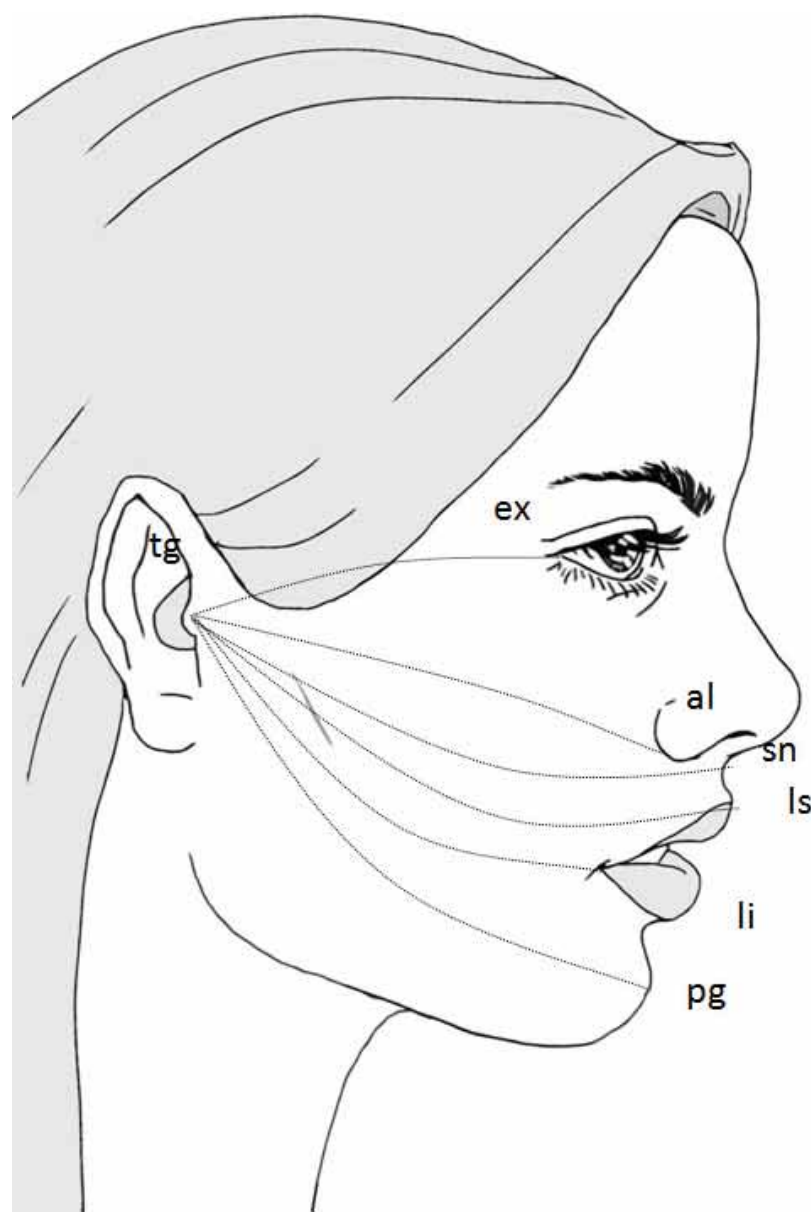

Fig. 2. Ubicación de los puntos antropométricos utilizados en el análisis facial de los 29 individuos, realizando las mediciones con hilo y posteriormente registradas sobre en una regla milimetrada (medidas volumétricas).

Posteriormente, el individuo se posicionó sentado en ángulo de $100^{\circ}$ en un sillón odontológico, manteniendo la posición de la cabeza con la visión en el plano horizontal, paralelo al piso. Las distancias analizadas correspondían a mediciones cutáneas que se originaban en el tragus y finalizaban en el exocantion, punto alar, punto subnasal, labio superior, labio inferior y pogonion (Fig. 2). La obtención de la distancia se obtuvo con un pie de metro digital marca Lionway ${ }^{\circledR}$ LME6278 0-150mm, precisión de $0.01 \mathrm{~mm}$, donde cada extremo del pie de metro se ubicaba en cada uno de los dos puntos, reflejando una medición en línea recta (Fig. 1); posteriormente se realizó la misma medición, enlazando los mismos puntos con un hilo de seda 4-0 que se instalaba sobre la piel. El hilo que registraba la medida se ubico sobre una regla milimetrada para obtener la medición final mediante la extensión del hilo sobre la regla (Fig. 2). Ambos elementos de medición (pie de metro e hilo) fueron posicionados sobre una

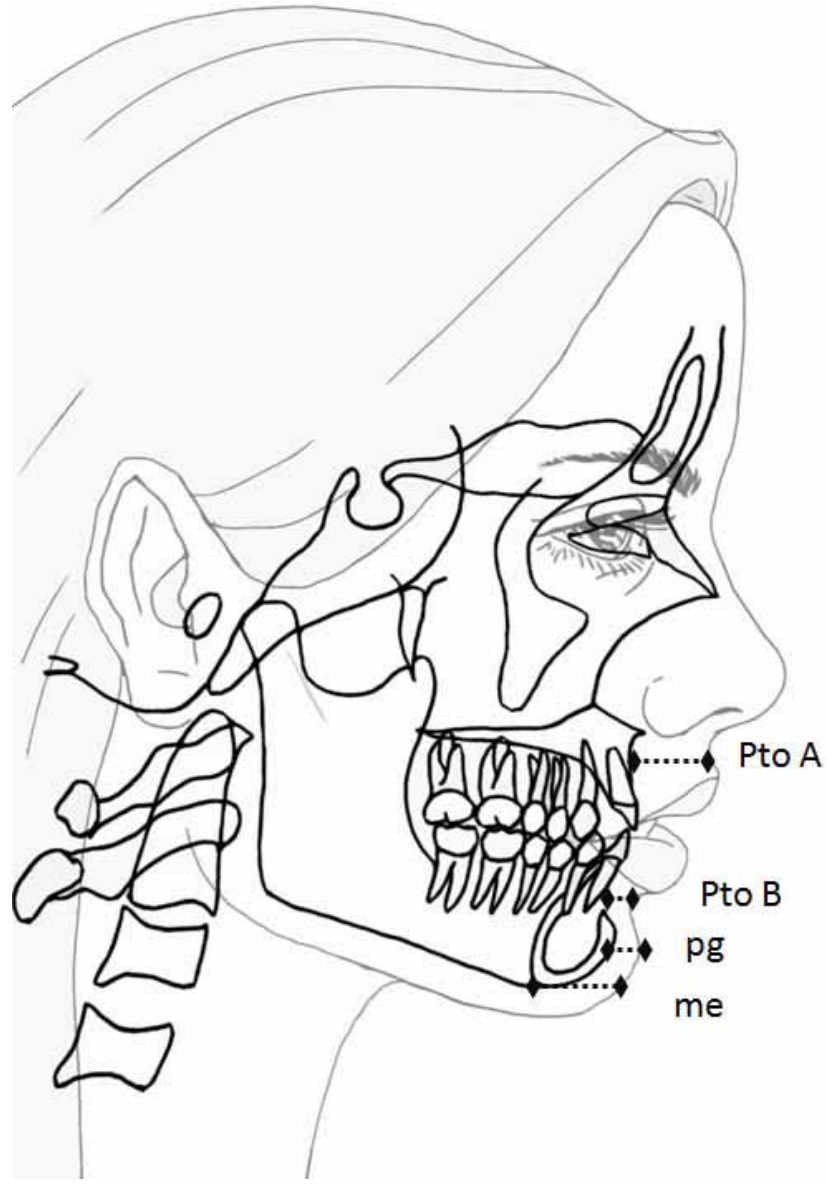

Fig. 3. Distancias entre tejido blando observadas en radiografía lateral de los 29 individuos de sexo femenino de entre 20 y 25 años. Las mediciones fueron realizadas paralelas al Plano de Frankfurt.

regla metálica milimetrada rígida marca Isofit $₫$ con precisión de $0.1 \mathrm{~mm}$ que finalmente arrojó la medida final. Para todas las medidas realizadas fue llevada a cabo una calibración intra e inter operador con el análisis de 10 sujetos, previo al comienzo de la toma de registros.

Posteriormente fueron realizadas las tomas radiográficas de tipo lateral (equipo Modelo: PantOs 16 Ceph. Panoramic \& Cephalometric Dental X-Ray Sistem. Marca Bluex® Año 2004, Assago-Italy), replicando la misma posición craneocervical expuesta en la etapa de análisis facial. Sobre esta imagen instalada en un negatoscopio de uso odontológico se realizo el análisis identificando el punto $A$, punto $B$, pogonion y mentón; las mediciones fueron de forma paralela al Plano de Frankfurt y registraron la distancia anteroposterior de los tejidos blandos presentes en la zona de análisis (Fig. 3). 
El análisis estadístico, fue llevado a cabo a través del software SPSS/PC+ versión 20.0, SPSS, Chicago, IL. Se realizó un análisis estadístico descriptivo para calcular distintos tipos de medidas. La prueba de análisis de varianza y el análisis de componentes principales se utilizaron para evaluar las diferencias entre las distintas variables. Para evaluar la distribución de normalidad se utilizó la prueba de ShapiroWilk. Se utilizó el coeficiente de correlación para cuantificar la correlación entre distintas medidas de variables continuas. El valor $p<0,05$ fue seleccionado para establecer significancia estadística.

\section{RESULTADOS}

Se estudiaron 29 mujeres de entre 20 y 25 años con una edad promedio de 22,4 años ( $\pm 1,2$ años). El peso obtenido registro un promedio de $57,6 \mathrm{~kg}( \pm 8,3$ $\mathrm{kg}$ ) y la talla presento un promedio de $1,6 \mathrm{mt}$; el IMC obtenido fue de 22,5, registrándose (según la clasificación propuesta por la OMS) 2 sujetos en peso inferior al normal, 20 con peso normal, 6 en sobrepeso y 1 con obesidad (Tabla I, Fig. 4).

Se observaron diferencias estadísticamente significativas cuando se compararon las variables de mediciones volumétricas (hilo sobre la piel) y las variables de mediciones rectas o no volumétricas (pie de metro en el punto inicial y final). Con el mismo análisis estadístico (t test) no se observo relación significativa entre el IMC y la distancia anteroposterior de tejidos blandos presentes en la radiografía lateral, obteniendo a nivel del punto $A p=0.107$, a nivel del punto $B$ $p=0.375$, a nivel del pogonion blando $p=0.124$ y a nivel del mentón $p=0.401$ (Tabla II).

Con un análisis de componentes principales se obtuvo la fuerza de asociación estadística entre el IMC

Tabla I. Caracterización de la muestra según edad e IMC.

\begin{tabular}{lcccc}
\hline & Promedio & $\begin{array}{c}\text { Desviación } \\
\text { Estándar }\end{array}$ & Mínimo & Máximo \\
\cline { 1 - 4 } Edad (años) & 22.4 & 1.2 & 20 & \\
Peso $(\mathrm{kg})$ & 57.6 & 8.3 & 44 & 76 \\
Altura $(\mathrm{m})$ & 1.60 & 0.047 & 1.47 & 1.69 \\
$\mathrm{IMC}\left(\mathrm{kg} / \mathrm{m}^{2}\right)$ & 22.5 & 3.2 & 18.1 & 31.2 \\
\hline
\end{tabular}

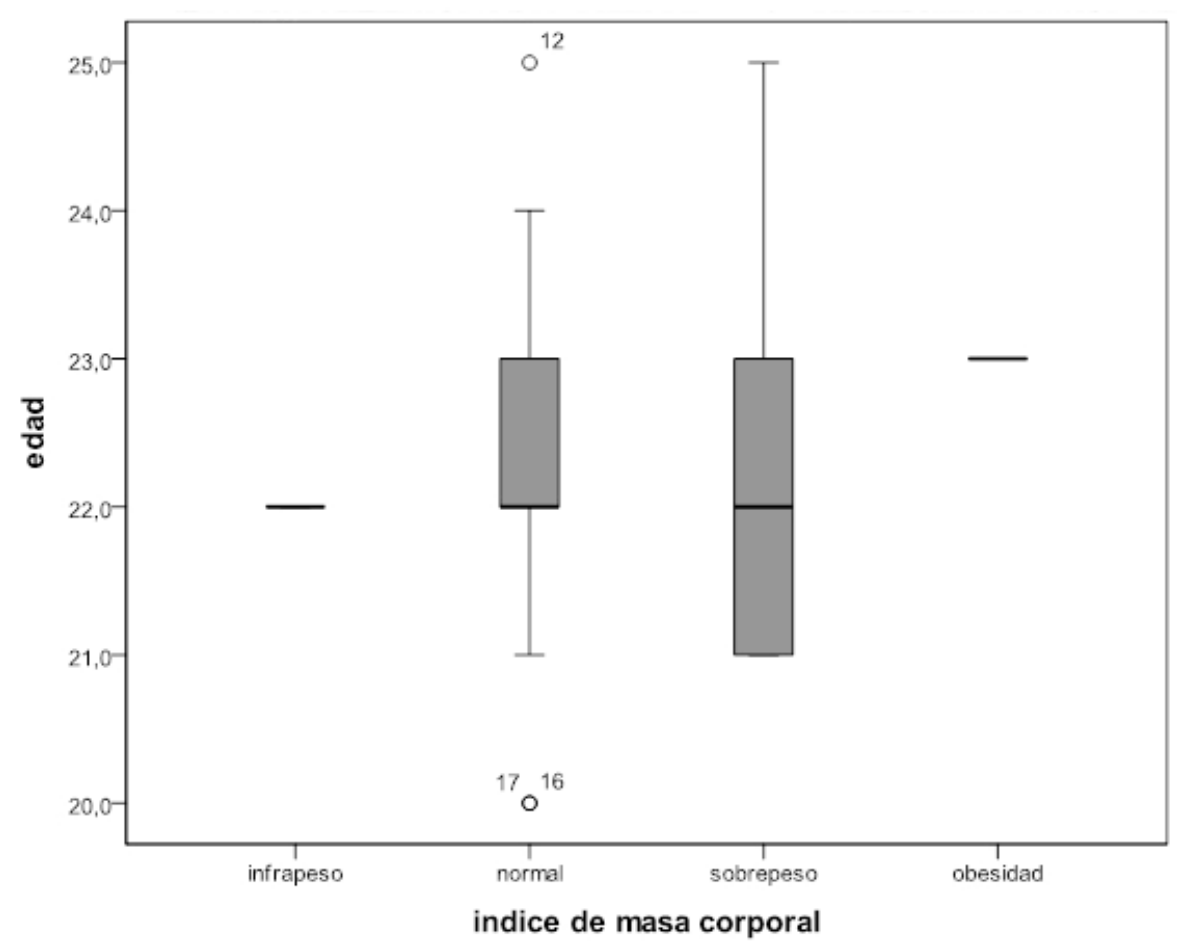

Fig. 4. Relación entre la edad y el IMC de los 29 sujetos del sexo femenino estudiados en esta investigación. 
y las variables estudiadas, demostrando que existía una relación significativa entre el IMC y la distancia tragus-ala nasal, tragus-cheilon, tragus-subnasal, tragus labio superior y tragus-labio inferior. Por otra parte, mediante estudio con ANOVA, el IMC no fue asociada a la percepción de la estética facial propia por parte de cada sujeto $(p=0.069)$.
Entre las medidas volumétricas (medidas con hilo) y las medidas no volumétricas (medidas con pie de metro) se realizaron comparaciones utilizando $t$ test, donde se encontró una asociadion estadísticamente significativa en las medidas tragus-cheilon derecho $(p=0.015)$, tragus-subnasal derecho $(p=0.004)$, tragus-subnasal izquierdo $(p=0.001)$, tragus-labio inferior $(p=0.023)$ y tragus-labio superior $(p=0.017)$.

Tabla II. Medidas de distancias volumétricas y rectas (no volumétricas) y la asociación entre ambas medidas a través del análisis $t$ test.

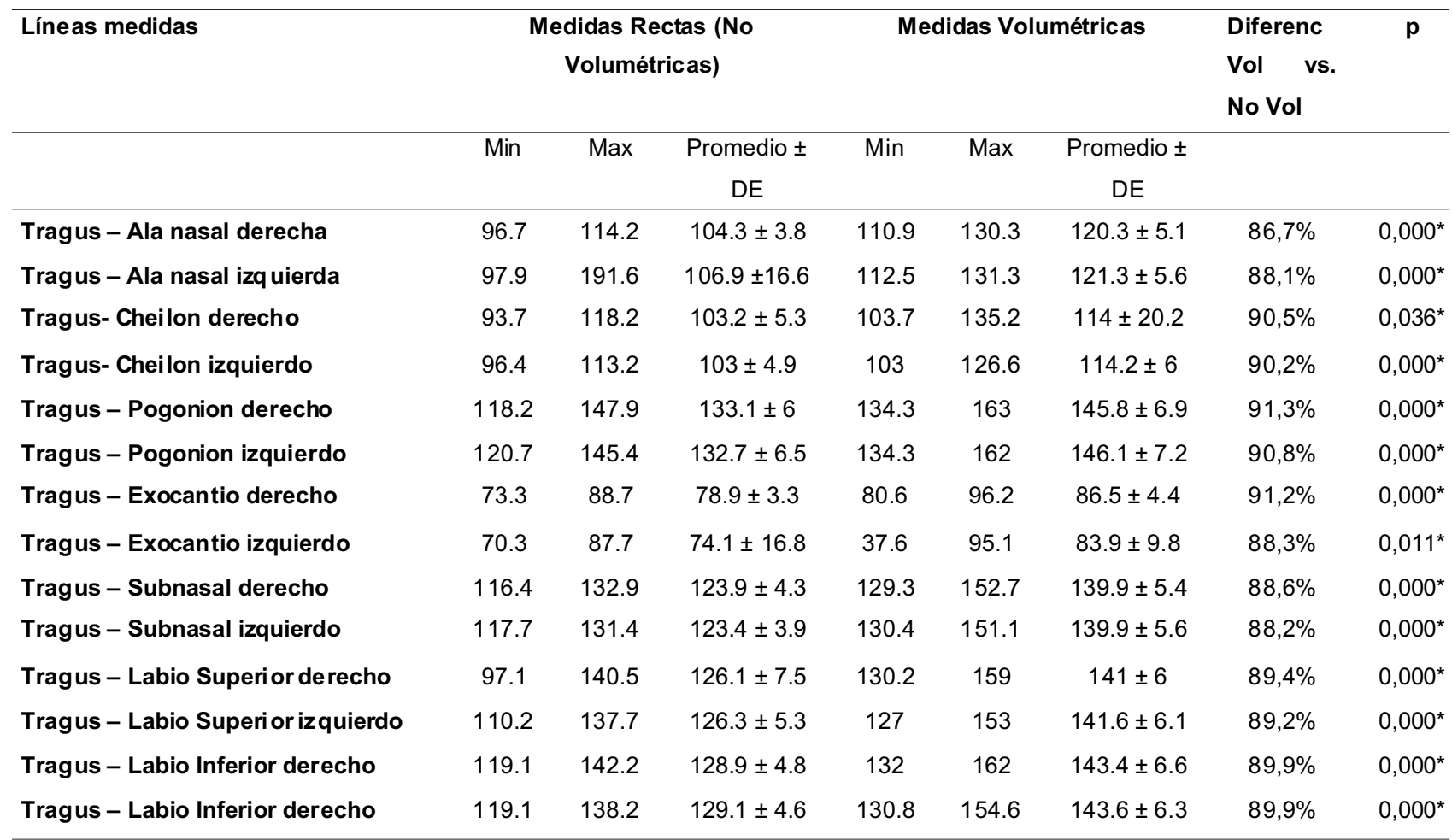

* Diferencias Significativa con nivel de significancia al 5\%.

\section{DISCUSIÓN}

Al evaluar la norma facial de 29 mujeres de entre 20 y 25 años se establecen criterios de proporciones faciales homogéneos y de fácil comparación. El ancho del tejido blando facial han sido estudiado comparando ambos géneros sin encontrar diferencias significativas (Domaracki \& Stephan 2006), con diferencias en promedios no mayor a 2,2 $\mathrm{mm}$. Por otra parte, Suazo et al. (2009) reportaron que el ancho de los tejidos blandos es más pronunciado y de mayor volumen en hombres que en mujeres. De la misma forma, Stephan et al. (2005), señalan que si bien existen diferencias significativas de ancho entre ambos sexos, estas son a menudo pequeñas o muy próximas.
Una evaluación morfológica objetiva y cualitativa previa a una cirugía facial es indispensable para los tratamientos quirúrgicos (kobayashi et al. 1990), de forma que los objetos de análisis, vistos en este articulo, son claramente necesarios de estudiar. Se han propuesto algunos métodos para medir y predecir el resultado de las modificaciones producidas por cirugías faciales (Ghoddousi et al., 2007), pero la deficiencia en análisis de volumen dificultan los hallazgos. Los análisis fotográficos fueron inicialmente utilizados con resultados variables (Kobayashi et al. 1990 \& Ghoddousi et al.), principalmente debido a los diferentes protocolos en la obtención de la imagen, cali- 
dad de la imagen, calidad de la cámara fotográfica, luminosidad, entre otras. Actualmente, nuevos estudios con análisis tridimensional y técnicas de análisis de software han revolucionado algunos aspectos del diagnóstico pre quirúrgico (Ghoddousi et al.; Dong et al., 2010), lo cual ha logrado mejorar las condicionantes terapéuticas y la predicción de resultados.

En las técnicas quirúrgicas faciales, se ha visto que pequeños cambios a nivel óseo pueden generar grandes cambios en tejido blando (Kobayashi et al.), por lo que movimientos en tejido duro deben ser realizados posterior a una rigurosa evaluación de los tejidos blandos.

En esta investigación, las medidas que siguieron el contorno facial realizadas con un hilo presentaron diferencias significativas con las medidas de los mismos lugares realizados de la forma recta sobre los mismos puntos antropométricos, de forma que es posible especular en que el tamaño de tejidos blandos presentes en el sector lateral de la cara influencia las mediciones realizadas; dicho de otra forma, el volumen del área del musculo bucal y el cuerpo adiposo de la mejilla o bola adiposa de bichat influenciarían los registros realizados.

Se podría especular entonces que la variación del plano adiposo intervendría en los resultados, de manera que el IMC fue relacionado significativamente con las medidas faciales revisadas, presentando diferencias significativas con las medidas realizadas sobre la piel. Este aspecto coincide con los estudios de Codinha, De Greef et al., (2009) y Dong et al., quienes encuentran asociaciones entre diferentes medidas antropométricas y el IMC. Así mismo, el Cuerpo Adiposo de la Mejilla se relaciona directamente con medidas de puntos antropométricos de la cara y con el IMC (Gravante \& Lo Grasso, 1997).

Los rasgos anatómicos varían de una población a otra (Husein et al., 2010); estos cambios se ven reflejados en diferencias entre medidas faciales en diferentes planos de medición, ya sea por cambios de niveles óseos distintos a cada población, o debido a la musculatura, o por el menor o mayor porcentaje de grasa que constituyen los tejidos para cada población o etnia (Simpsons \& heneberg, 2002). Cirugías de tejidos blandos que envuelven planos adiposos y musculares finalizan con resultados estéticos positivos (Xu \& Yu, 2013).

Basándose en los resultados encontrados en nuestro estudio y además en lo propuesto por De Greef et al., se sugiere que el IMC tiene un rol importante en las características faciales; el aumento del IMC se ha asociado frecuentemente con la disminución de autoestima, sin embargo algunos autores proponen que no hay diferencias significativas entre los individuos cuando se estudia la variable IMC con relación a la autoestima (Aguilar et al., 2002). Sin embargo, otros han sugerido que al aumentar el IMC existe la probabilidad de aumentar el grado de insatisfacción corporal del individuo (Casillas et al., 2006).

Finalmente, se puede concluir que el IMC presenta influencias en la morfología facial, estableciendo diferencias en el volumen de los tejidos faciales. El IMC no influencia en el ancho de tejidos blandos visto en radiografías laterales.

HUENTEQUEO-MOLINA, C.; NAVARRO, P.; OLATE, S. Body Mass index influence in facial pattern of young women. Preliminary results. Int. J. Odontostomat., 7(3):407-414, 2013.

ABSTRACT: The position and volume of the face soft-tissue yield the necessity of a precise plan at the facial surgery time; the influence of the adipose tissue on these characteristics can be present. The aim of this study is to identify the volumetric and non-volumetric measures of anthropometrics facial points and his relationship with the Body Mass Index (BMI). It was been analyzed 29 female subjects between 20 and 25 years old (mean 22.5 years). It was include subjects with self-perception of facial normalcy and without conditions associated to trauma, facial surgery or orthodontic treatment. It was made a sequence of facial analysis measuring with a thread over the face and a caliper the same points, studying distances between anthropometrics points previous define. Previously, it was made a study of the weight and size of the subjects, through standards conditions. At the third stage it was made lateral radiographs to identify the soft tissue present at the superior and inferior lip region, point $A$, point $B$ and $P$ ogonion. The data analysis was made with the t test of relationship samples, considering a $p$ value $\leq 0.05$; the statistics analysis ANOVA was used to define the influence of the BMI over the variables. The weight registered a mean of $57.7 \mathrm{~kg}( \pm 8,3 \mathrm{~kg})$ and the size performed a mean of $1.6 \mathrm{~m}$.; the BMI acquired was 22.5, registering, being 2 subjects considerate inferior to the normal weight, 6 overweight and 1 obesity. It was seem that the volumetric variables were different to volumetric variables of the face and they were associated significantly with the IMC. The IMC didn't show positive relationship with the measures made on the laterals radiographies; finally, the BMI, as well, did not associate to the self-perception of facial aesthetic $(p=0.069)$. It is possible to conclude that the BMI influence the measures obtained on the face and did not influence on the register made over the laterals radiographs.

KEY WORD: Body mass index, Facial esthetic, facial surgery 


\section{REFERENCIAS BIBLIOGRÁFICAS}

Aguilar, A.; Puig, P.; Luna, L.; Sánchez, P.; Rodríguez, R. \& Rodríguez, L. La autoestima y su relación con el índice de masa corporal al culminar la adolescencia. Rev. Mex. Pediatr., 69(5):190-3, 2002.

Alcalde, R.; Jinno, T.; Orsini, M.; Sasaki, A.; Sugiyama, R. \& Matsumura, T. Soft tissue cephalometric norms in Japanese adults. Am. J. Orthod. Dentofacial Orthop., 118(1):84-9, 2000.

Casillas, M.; Montaño, N.; Reyes, V.; Bacardí, M. \& Jiménez, A. A mayor IMC mayor grado de insatisfacción de la imagen corporal. Rev. Biomed., 17: 243-9, 2006.

Codinha S. Facial soft tissue thicknesses for the Portuguese adult population. Forensic Sci. Int., 184(1-3): 80e1-80e7, 2009.

De Greef, S.; Vandermeulen, D.; Claes, P.; Suetens, P. \& Willems, $G$. The influence of sex, age and body mass index on facial soft tissue depths. Forensic Sci. Med. Pathol., 5(2):60-5, 2009.

Domaracki, M., \& Stephan, C. N. Facial Soft Tissue Thicknesses in Australian Adult Cadavers. J. Forensic Sci., 51(1):5-10, 2006.

Dong, Y.; Huang, L.; Feng, Z.; Bai, S.; Wu, G. \& Zhao, Y. Influence of sex and body mass index on facial soft tissue thickness measurements of the northern Chinese adult population. Forensic Sci. Int., 222(1-3):396e1-396e7, 2012.

Dong, Y.; Zhao, Y.; Bai, S.; Wu, G. \& Wang, B. Three-dimensional anthropometric analysis of the Chinese nose. J.P.R.A.S., 63(11):1832-9, 2010.

Douglas, T. S. Image processing for craniofacial landmark identification and measurement: a review of photogrammetry and cephalometry. Comput. Med. Imaging Graph., 28(7): 401-9, 2004.

Farkas, L.G; Hreczko, T. \& Kolar, J. Vertical and horizontal proportions of the face in young adult North American caucasians: Revision of neoclassical canons. Plast. Reconstr. Surg. 75: 328-338, 1985.

Farkas, L.G; Katic, M. \& Forrest, C.R. International anthropometric study of facial morphology in various ethnic groups/races. J. Craniofac. Surg., 16:615-46, 2005.

Gassmann, C.; Nishioka, G.; Van Sickels, J. \& Thrash, W. A lateral cephalometric analysis of nasal morphology following Le Fort I osteotomy applying photometric analysis techniques. J. Oral Maxillofac. Surg., 47(9):92630, 1989.

Ghoddousi, H.; Edler, R.; Haers, P.; Wertheim, D. \& Greenhill, D. Comparison of three methods of facial measurement. Int. J. Oral Maxillofac. Surg., 36: 250-8, 2007.

Godt, A.; Bechtold, T.; Schaupp, E.; Zeyher, C.; Koos, B.; Baas, E. \& Berneburg, M. Correlation between occlusal abnormalities and parameters investigated by three-dimensional facial photography. Angle Orthod., 83:782-9, 2013.

Gravante, G. \& Lo Grasso, S. Bichat's fat-pad: correlations with some anthropometric parameters. Ann. Hum. Biol., 24(2):181-5, 1997.

Husein, O.; Sepehr, A.; Garg, R.; Sina-Khadiv, M.; Gattu, S., Waltzman, J.; Wu, E.; Shieh, M.; Heitmann, G. \& Galle, S. Anthropometric and aesthetic analysis of the Indian American woman's face. J.P.R.A.S., 63(11):1825-31, 2010.

Kobayashi, T.; Ueda, K. \& Honmd, K. Three-Dimensional Analysis of Facial Morphology before and after Orthognathic Surgery. J. Craniomaxillofac. Surg., 18:6873, 1990.

Olszewski, R.; Zech, F.; Cosnard, G.; Nicolas, V.; Macq, B.; \& Reychler, H. Three-dimensional computed tomography cephalometric craniofacial analysis: experimental validation in vitro. Int. J. Oral Maxillofac. Surg., 36:82833, 2007.

Reddy, P.; Kashyap, B.; Hallur, N. \& Sikkerimath, B. Advancement genioplasty--cephalometric analysis of osseous and soft tissue changes. J. Maxillofac. Oral Surg., 10(4):288-95, 2011.

Sahni, D.; Sanjeev; Singh, G.; Jit, I. \& Singh, P. Facial soft tissue thickness in northwest Indian adults. Forensic Sci. Int., 176(2-3):137-46, 2008.

Simpson, E. \& Henneberg, M. Variation in soft-tissue thicknesses on the human face and their relation to craniometric dimensions. Am. J. Phys. Anthropol., 118(2):121-33, 2002.

Sipahiog lu, S.; Ulubay, H. \& Diren, H.B. Facial soft tissue thickness database of Turkish population: MRI study. Forensic Sci. Int. 219(1-3):282e1-282e8, 2012.

Starck, W. \& Epker, BN. Cephalometric analysis of profile nasal esthetics. Part I. Method and normative data. Int. J. Adult Orthodon. Orthognath. Surg., 11(2):91-103, 1996. 
HUENTEQUEO-MOLINA, C.; NAVARRO, P.; OLATE, S. Influencia de índice de masa corporal en las características faciales de mujeres jóvenes. Resultados preliminares. Int. J. Odontostomat., 7(3):407-414, 2013.

Stephan, C.N.; Norris, R.M. \& Henneberg, M. Does sexual dimorphism in facial soft tissue depths justify sex distinction in craniofacial identification. J. Forensic Sci., 50(3):513-8, 2005.

Suazo, I.C.; Perez, F.J. \& Torres S.R. Tissue Tickness in spanish corpses and your applications on the medicolegal identification. Int. J. Morphol., 25(1): 109-16, 2007.

Uysal, T.; Baysal, A.; Yagci, A. \& Sigler, L.M.; McNamara, J.A. Jr. Ethnic differences in the soft tissue profiles of Turkish and European-American young adults with normal occlusions and well-balanced faces. Eur. J. Orthod., 34(3):296-301, 2012

$X u$, J. \& Yu, Y. A modified surgical method of lower-face recontouring. Aesthetic Plast. Surg., 37(2): 216-21, 2013.
Correspondencia:

Prof. Dr. Sergio Olate

Unidad de Cirugía Oral y Maxilofacial

Universidad de La Frontera

Claro Solar No 115

Temuco

CHILE

Tel: +56 (45) 2325000

Email: sergio.olate@ufrontera.cl

Recibido : 22-02-2013

Aceptado: 26-08-2013 